\title{
Communication
}

\section{Phenolic Polyketides from the Co-Cultivation of Marine-Derived Penicillium sp. WC-29-5 and Streptomyces fradiae 007}

\section{Yi Wang ${ }^{1}$, Liping Wang ${ }^{1}$, Yibin Zhuang ${ }^{1}$, Fandong Kong ${ }^{1}$, Cuixian Zhang ${ }^{2}$ and Weiming Zhu ${ }^{1, *}$}

1 Key Laboratory of Marine Drugs, Ministry of Education of China, School of Medicine and Pharmacy, Ocean University of China, Qingdao 266003, China; E-Mails: wangyi0213@hotmail.com (Y.W.); lipingw2006@163.com (L.W.); zhuang_yb@tib.cas.cn (Y.Z.); kongfandong501@126.com (F.K.)

2 School of Chinese Material Medica, Guangzhou University of Chinese Medicine, Guangzhou 510006, China; E-Mail: zhangcuixian@yahoo.com.cn

* Author to whom correspondence should be addressed; E-Mail: weimingzhu@ouc.edu.cn; Tel./Fax: +86-532-8203-1268.

Received: 15 January 2014; in revised form: 24 February 2014 / Accepted: 21 March 2014 / Published: 4 April 2014

\begin{abstract}
Penicillium sp. WC-29-5 was co-cultured with Streptomyces fradiae 007 to produce five natural products $(\mathbf{1}-\mathbf{3}, \mathbf{4 a}$ and $\mathbf{4 b})$ that were isolated and characterized by spectroscopic analysis. Interestingly, these compounds were found to be different from those produced in discrete fungal and bacterial controls. Among these compounds, the absolute configurations of compounds $\mathbf{4 a}$ and $\mathbf{4 b}$ were determined for the first time by X-ray single crystal diffraction experiments and electronic circular dichroism (ECD) calculations. An evaluation of the cytotoxic activities of these compounds revealed that $\mathbf{4 b}$ was moderately cytotoxic towards HL-60 and H1975 tumor cells with $\mathrm{IC}_{50}$ values of 3.73 and $5.73 \mu \mathrm{M}$, respectively, whereas compound 4a was only moderately cytotoxic towards $\mathrm{H} 1975$ cells with an $\mathrm{IC}_{50}$ value of $3.97 \mu \mathrm{M}$.
\end{abstract}

Keywords: Penicillium sp.; Streptomyces fradiae; co-cultivation; secondary metabolites; cytotoxicity 


\section{Introduction}

Natural products have proven to be an incredible resource for drug discovery [1] and the microorganisms responsible for the production of natural products can be considered in some respects as a type of renewable and eco-friendly resource. Many of the microbial gene clusters involved in the synthesis of natural products, however, are silent under traditional lab conditions [2-4], and significant research efforts have consequently been devoted to developing new strategies and approaches for activating silent gene clusters to increase the diversity and availability of microbial natural products. It is generally accepted that microbial natural products are used by microbes to communicate with each other, to protect their hosts, or to inhibit the growth of other competitive species in their natural environment [3]. From the perspective of cooperative interactions, the co-cultivation or mixed fermentation of two or more microbes represents a promising approach for simulating physiological conditions and activating silent gene clusters [3,5-7]. As part of our ongoing research towards identifying new bioactive natural products from marine-derived microorganisms [8-10], we recently became interested in evaluating the mixed fermentation of two microbes and found that the co-cultivation of Penicillium sp. WC-29-5 and Streptomyces fradiae 007 led to the production different metabolites to those produced by each species in isolation (Figure 1). Streptomyces fradiae is well known for its production of several antibiotics, including neomycin and fosfomycin [11,12]. We previously reported that $S$. fradiae 007 could produce staurosporine [13], and the three indolocarbazoles, fradcarbazoles A-C [14]. Herein, we report the isolation, structural elucidation and evaluation of the cytotoxicity of the metabolites produced by the co-cultivation of Penicillium sp. WC-29-5 and S. fradiae 007.

Figure 1. HPLC profiles of secondary metabolites from S. fradiae 007; Penicillium sp. WC-29-5 and co-cultivation of two strains from up to down (HPLC eluent: 0-30 min, $50 \% \mathrm{MeOH}$; flow rate: $1 \mathrm{~mL} / \mathrm{min}$; detection wavelength: $202 \mathrm{~nm}$ ).
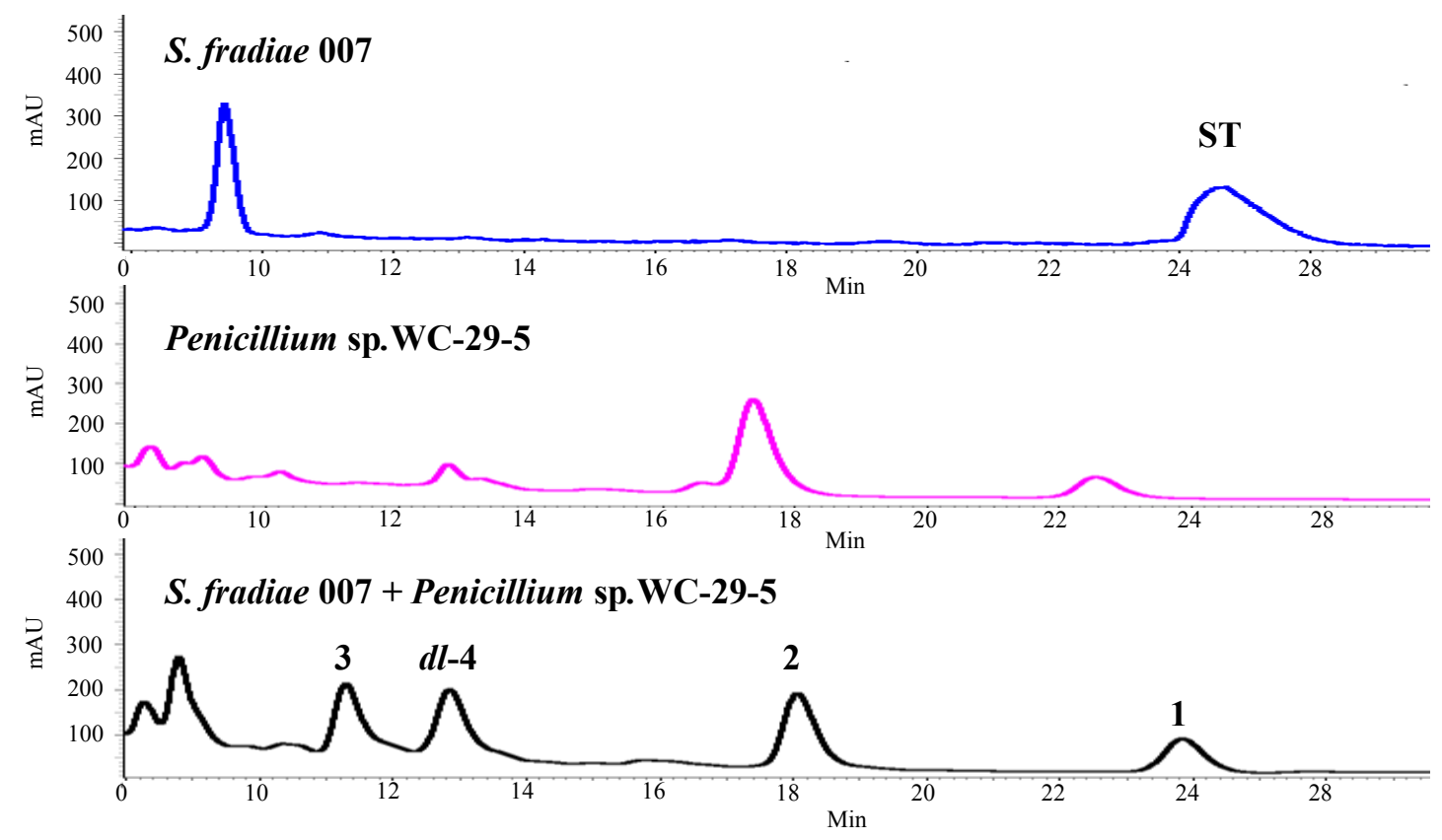


\section{Results and Discussion}

Penicillium sp. WC-29-5 and S. fradiae 007 were co-cultivated in the same medium in the current study to induce the production of different bioactive compounds as a consequence of the competitive nature of the environment. The products formed during the co-cultivation process were investigated at different mix-times. S. fradiae 007 and Penicillium sp. WC-29-5 were cultivated in isolation at $28{ }^{\circ} \mathrm{C}$ on a $160 \mathrm{rpm}$ shaker. Seeds of the $S$. fradiae 007 (10\% inoculum) of different ages (i.e., 0 to 5 day old) were mixed with the same inoculum size of Penicillium sp. WC-29-5, and the resulting co-cultures were incubated under the same conditions for 7 days. The results revealed that the growing states and secondary metabolites of the co-cultivated species were the same as those observed for the culturing of Penicillium sp. WC-29-5 in isolation when the two strains were seeding at the same time (0 day). In contrast, the growing states and the secondary metabolites observed in the co-cultivation experiments were more like those observed for the culturing of $S$. fradiae 007 in isolation when WC-29-5 was added into the 3-day, 4-day and 5-day old cultures of 007, respectively. Significant variations were observed in the secondary metabolites following the seeding of WC-29-5 into a 2-day old culture of 007 (Figure 1). Based on these results, the co-fermentation process was performed by mixing Penicillium sp. WC-29-5 with a 2-day old culture of $S$. fradiae 007 . The resulting mixture was then incubated for 7 days on a $160 \mathrm{rpm}$ shaker at $28{ }^{\circ} \mathrm{C}$. The whole co-fermentation broth $(15 \mathrm{~L})$ was extracted thoroughly with ethyl acetate (EtOAc) and the EtOAc extracts were isolated and purified by chromatography, including HPLC, to yield five compounds. By means of spectroscopic analysis, X-ray single crystal diffraction, and theoretical calculations of the electronic circular dichroism (ECD), we have successfully identified these compounds as deoxyfunicone (1) [15], alternariol (2) [16], vermistatin (3) [17], (9R,14S)-epoxy-11-deoxyfunicone (4a), and (9S,14R)-epoxy-11-deoxyfunicone (4b) (Figure 2).

Figure 2. Compounds from the co-cultivation of Penicillium sp. WC-29-5 and S. fradiae 007 .

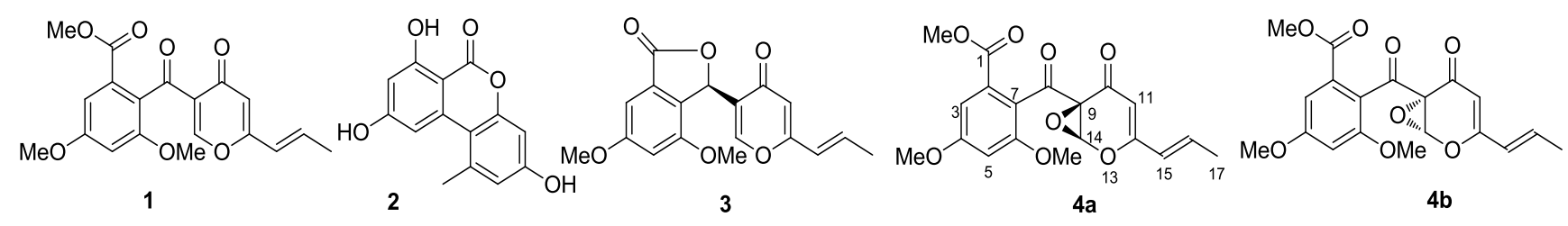

Compounds $\mathbf{4 a}$ and $\mathbf{4 b}$ were initially obtained as a mixture, and analysis by HRESIMS revealed a peak with an $m / z$ value of $397.0902[\mathrm{M}+\mathrm{Na}]^{+}$, which corresponded to a molecular formula of $\mathrm{C}_{19} \mathrm{H}_{18} \mathrm{O}_{8}$. Consideration of the literature revealed that the NMR data for this mixture were similar to those reported for 9,14-epoxy-11-deoxyfunicone (Supplementary Information, Table S1, Figures S1-S7), and indicated that compounds $\mathbf{4 a}$ and $\mathbf{4 b}$ were based on the same planar structure [17]. The results of analysis by X-ray single crystal diffraction (Figure 3) also supported this suggestion. The mixture of compounds $\mathbf{4 a}$ and $\mathbf{4 b}$ gave a large Rw (absolute configuration parameter) value and a zero value for its specific rotation, which indicated that these compounds were a racemic mixture. Subsequent analysis by chiral chromatography revealed the presence of two peaks, which were separated to give optically pure 4a and 4b (Figure 4). Compounds 4a and 4b showed equal and opposite specific 
rotations (i.e., $[\alpha]^{23}{ }_{\mathrm{D}}+15.8$ and -15.4 , respectively), which indicated that they were a pair of enantiomers. The CD curves of compounds $\mathbf{4 a}$ and $\mathbf{4 b}$ were measured to determine their absolute configurations, and their ECD curves were also calculated using the time-dependent density functional theory (TD-DFT) method at the B3LYP/6-31G(d) level [18]. Preliminary conformational distribution searches were performed using version 7.5 of the HyperChem software, and the corresponding minimum geometries were fully optimized using DFT at the B3LYP/6-31G(d) level using the Gaussian 03 program package. The results showed that the measured CD curves for $\mathbf{4 a}$ and $\mathbf{4 b}$ were in good agreement with the calculated ECD curves for $(9 R, 14 S)-\mathbf{4}$ and $(9 S, 14 R)-\mathbf{4}$, respectively (Figure 5). Furthermore, the CD Cotton effects observed for $\mathbf{4 a}$ and $\mathbf{4 b}$ were attributed to exciton coupling between the transition dipoles of the benzoyl and hexa-2,4-dien-1-one moieties (chromophores). The CD Cotton effect at a long-wave length could be used to predict the absolute configuration according to the Harada-Nakanishi non-empirical rule [19,20]. The positive long-wave Cotton effect of $\mathbf{4 a}$ at $\lambda_{\max } 336 \mathrm{~nm}$ indicated that the front chromophore existed in a clockwise arrangement relative to the rear chromophore (Figure 6), and implied that compound 4a was in the $(9 R, 14 S)$-configuration. The bisignate $\mathrm{CD}$ Cotton effect of $\mathbf{4 b}$ indicated that this compound existed in the opposite configuration to that of $\mathbf{4 b}$. Thus, compounds $\mathbf{4 a}$ and $\mathbf{4 b}$ were ambiguously determined to be $(9 R, 14 S)$ - and $(9 S, 14 R)$-epoxy-11-deoxyfunicone, respectively.

Figure 3. The X-ray crystal structure of the racemic $d l-4$.

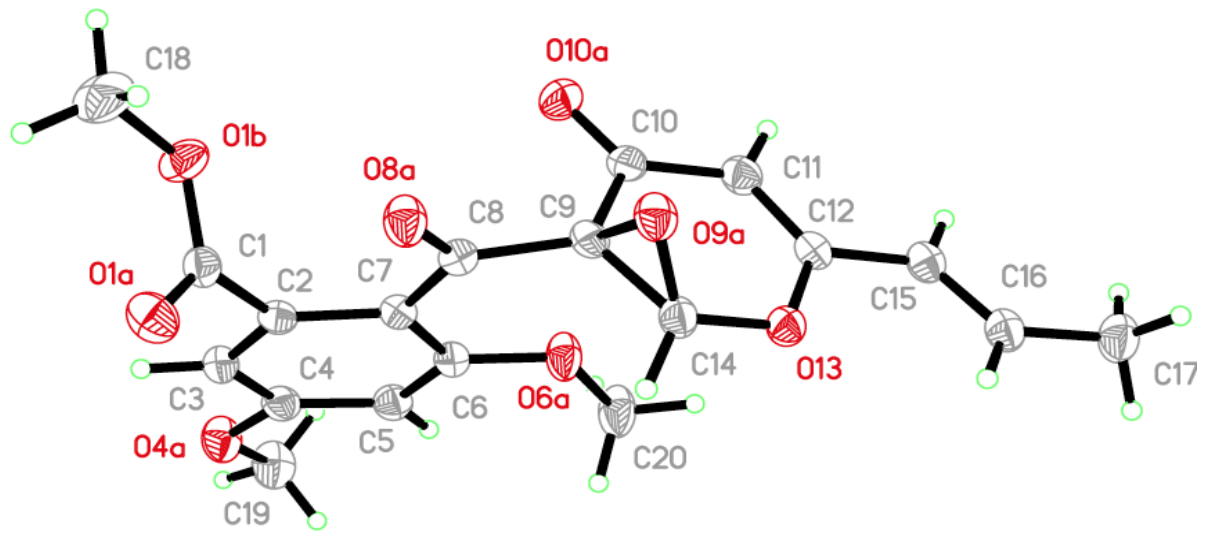

Figure 4. The chiral HPLC analysis and resolution of the racemic $d l-4$ by a Chiralpak IC column (n- $\left.\mathrm{C}_{6} \mathrm{H}_{14}-\mathrm{EtOH} 20: 80\right)$.

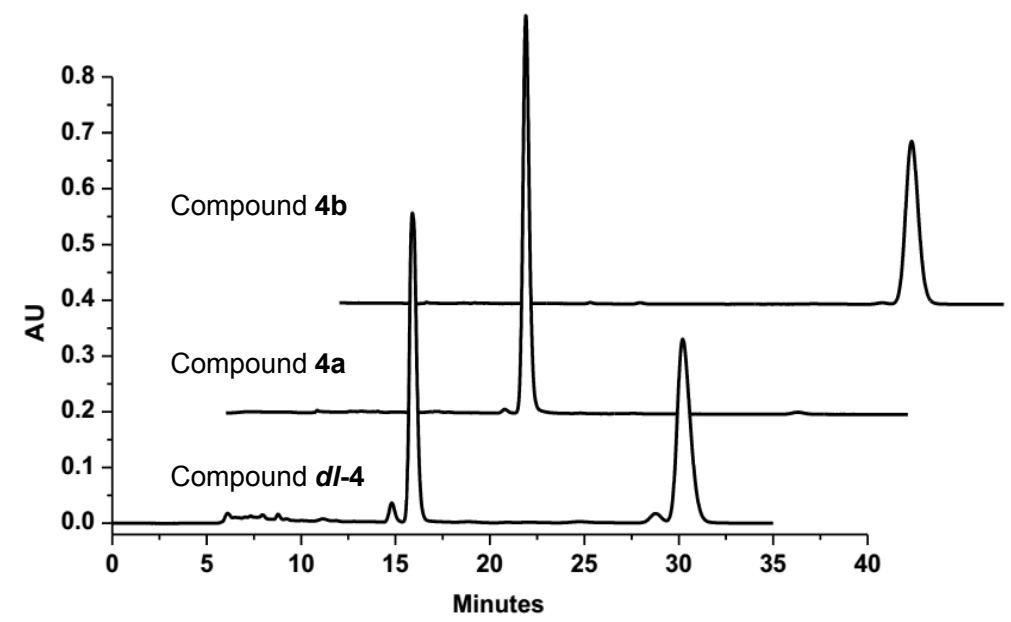


Figure 5. The measured and calculated electronic circular dichroism (ECD) curves of compounds $\mathbf{4 a}$ and $\mathbf{4 b}$.

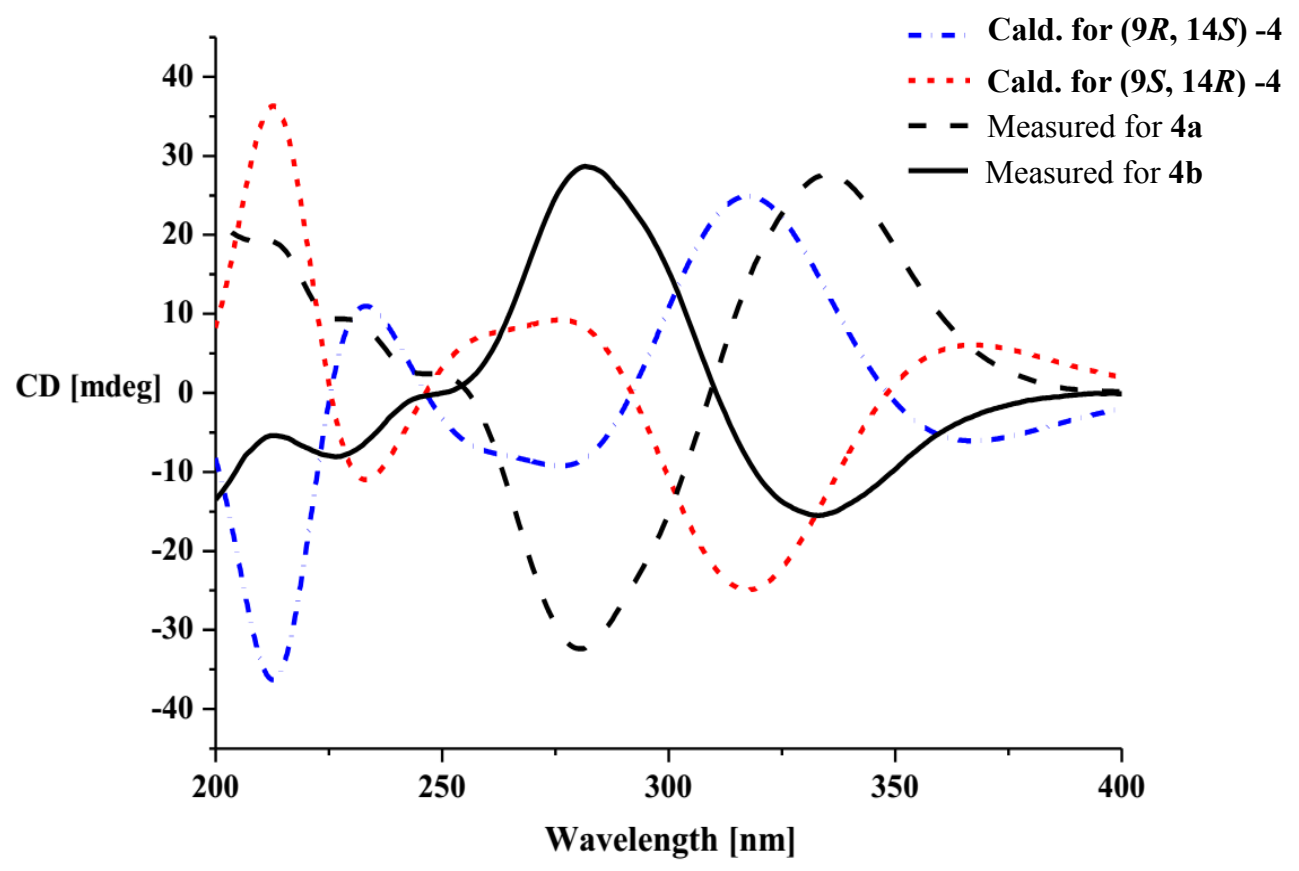

Figure 6. Stereo view of $4 a$ (bold lines denote the electric transition dipole of the chromophores).

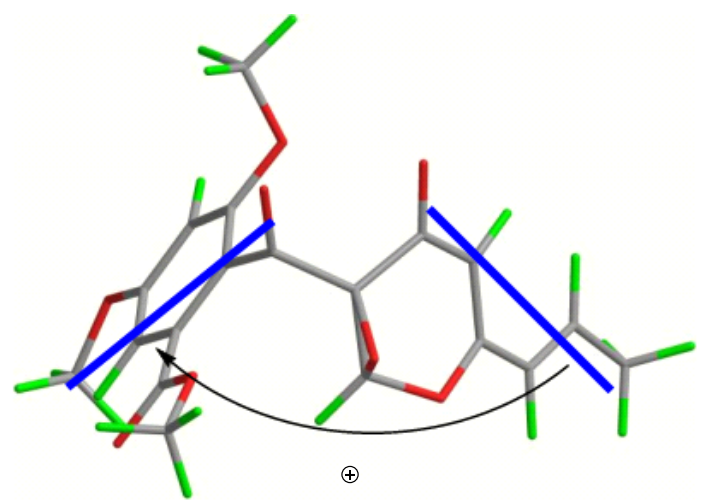

Stereo-configuration unresolved 9,14-epoxy-11-deoxyfunicone has previously been isolated from the culture of Talaromyces flavus IFM52668 and has been reported to be active against Aspergillus niger with a $10-\mathrm{mm}$ inhibition zone at $200 \mu \mathrm{g} / \mathrm{disc}$ [17]. To the best of our knowledge, however, there have been no reports in the literature pertaining to the cytotoxicity of this compound towards tumor cell lines. In the current study, we have evaluated the cytotoxicities of optically pure compounds $\mathbf{3}, \mathbf{4 a}$ and $\mathbf{4 b}$ against HL-60 and H1975 cells using the MTT method. The result of the MTT assay showed that the $d$-isomer 4a was only moderately active against $\mathrm{H} 1975$ tumor cells with an $\mathrm{IC}_{50}$ value of $3.97 \mu \mathrm{M}$, whereas the $l$-isomer $\mathbf{4 b}$ was moderately active against both two tumor cell lines with $\mathrm{IC}_{50}$ values of 3.73 and $5.73 \mu \mathrm{M}$, respectively. Compound $\mathbf{3}$ did not show any cytotoxic activity towards the two tumor cell lines $\left(\mathrm{IC}_{50}>10 \mu \mathrm{M}\right)($ Table 1$)$. 
Table 1. Cytotoxicities of compounds 3, 4a and $\mathbf{4 b}$ against HL-60 and H1975 cells.

\begin{tabular}{cccc}
\hline \multirow{2}{*}{ Cells } & \multicolumn{3}{c}{$\mathbf{I C}_{\mathbf{5 0}}(\boldsymbol{\mu M})$} \\
\cline { 2 - 4 } & $\mathbf{3}$ & $\mathbf{4 a}$ & $\mathbf{4 b}$ \\
\hline HL-60 & $>10$ & $>10$ & 3.73 \\
H1975 & $>10$ & 3.97 & 5.73 \\
\hline
\end{tabular}

S. fradiae 007 and Penicillium sp. WC-29-5 were incubated separately under the same conditions to those used for the co-cultivation. The co-cultivation process afforded different secondary metabolites to those obtained from the individual fermentation processes (Figure 1). ST, which is the major product of obtained from the cultivation of $S$. fradiae 007, was not found in the co-cultivation experiment. Furthermore, compounds 1, 2, 4a and $\mathbf{4 b}$ were only produced in the co-cultivation of S. fradiae 007 with Penicillium sp. WC-29-5. These results suggested that the co-cultivation process was activating silent gene clusters to produce different secondary metabolites.

\section{Experimental Section}

\subsection{General Experimental Procedures}

Specific rotations were obtained on a JASCO P-1020 digital polarimeter. UV spectra were recorded on a Beckman DU 640 spectrophotometer. IR spectra were obtained on a Nicolet NEXUS 470 spectrophotometer as $\mathrm{KBr}$ disks. ${ }^{1} \mathrm{H}$ and ${ }^{13} \mathrm{C}$ NMR analyses, including DEPT spectra and 2D NMR experiments, were recorded on a JEOL JNMECP 600 spectrometer using TMS as internal reference standard. The chemical shifts for the NMR spectra were recorded as $\delta$ values in ppm. HRESIMS was conducted on a Q-TOF Ultima Global GAA076 LC mass spectrometer (Waters Asia, Ltd., Singapore). CD spectra were measured on a JASCO J-815 spectropolarimeter (Jasco Corporation, Tokyo, Japan). Semipreparative HPLC (Waters Corporation, Milford, MA, USA) was performed using an ODS column (YMC-pack ODS-A, $10 \times 250 \mathrm{~mm}, 5 \mu \mathrm{m}$ ) with a flow rate of $4 \mathrm{~mL} / \mathrm{min}$. HPLC was performed using an ODS column (YMC-pack C18, $4.6 \times 250 \mathrm{~mm}, 5 \mu \mathrm{m}$ ) with a flow rate of $2 \mathrm{~mL} / \mathrm{min}$. TLC and column chromatography (CC) were performed on plates precoated with silica gel GF254 $(10-40 \mu \mathrm{m})$ and over silica gel (200-300 mesh, Qingdao Marine Chemical Factory, Qingdao, China) and Sephadex LH-20 (Amersham Biosciences, Uppsala, Sweden), respectively.

\subsection{Microbial Materials}

The fungus Penicillium sp.WC-29-5 was isolated from the mangrove soil around the roots of Aegiceras corniculatum, Wenchang, Hainan Province of China. It was identified according to its morphological characteristics and 18S rRNA gene sequences (Genbank access No. KJ138167). Streptomyces fradiae 007 were isolated and identified from a sediment sample collected in Jiaozhou Bay, Shandong Province of China $[13,14]$. The voucher specimens were deposited in our laboratory at $-80^{\circ} \mathrm{C}$. 


\subsection{Fermentation and Extraction}

S. fradiae 007 and Penicillium sp. WC-29-5 were separately cultured on an orbital shaker (160 rpm) at $28{ }^{\circ} \mathrm{C}$ for 2 days in $500-\mathrm{mL}$ conical flasks containing the liquid medium (100 mL/flask, $\left.\mathrm{pH} 6.5\right)$, which was composed of glucose $(10 \mathrm{~g} / \mathrm{L})$, maltose $(20 \mathrm{~g} / \mathrm{L})$, mannitol $(20 \mathrm{~g} / \mathrm{L})$, monosodium glutamate $(10 \mathrm{~g} / \mathrm{L})$, yeast extract $(3 \mathrm{~g} / \mathrm{L}), \mathrm{KH}_{2} \mathrm{PO}_{4}(0.5 \mathrm{~g} / \mathrm{L}), \mathrm{MgSO}_{4} \cdot 7 \mathrm{H}_{2} \mathrm{O}(0.3 \mathrm{~g} / \mathrm{L})$ and sea water. Penicillium sp. WC-29-5 was then seeded into the $S$. fradiae 007 cultures of in 500-mL conical flasks $(100 \mathrm{~mL} / \mathrm{flask}$, $\mathrm{pH} 6.5)$ at $5 \%$ of the final concentration for each strain, and the resulting mixtures were co-cultured for 7 days under the same conditions.

The totally cultivated medium $(15 \mathrm{~L})$ was harvested and filtered through cheesecloth to separate the broth from the mycelia. The broth was concentrated under reduced pressure to about $5 \mathrm{~L}$ and then extracted three times with EtOAc to give an EtOAc solution, whereas the mycelia was extracted three times with acetone. The acetone solution was concentrated under reduced pressure to afford an aqueous solution, which was extracted three times with EtOAc to give a second EtOAc solution. Both EtOAc solutions were combined and concentrated under reduced pressure to give an EtOAc extract (20.0 g). S. fradiae 007 and Penicillium sp. WC-29-5 were also incubated separately under the same conditions as controls. The chemical diversities of the secondary metabolites obtained from the different EtOAc extracts were investigated by HPLC.

\subsection{Purification and Identification}

The EtOAc extract (20.0 g) was separated into nine fractions (fractions 1-9) by CC over silica gel using a stepwise gradient eluting with mixtures of petroleum ether and $\mathrm{CHCl}_{3}(0 \%-100 \%$, v/v) and $\mathrm{CHCl}_{3}$ and $\mathrm{MeOH}(0 \%-100 \%, \mathrm{v} / \mathrm{v})$. Fraction 6 was separated into seven fractions (fractions 6-1 to 6-7) by $\mathrm{CC}$ over silica gel eluting with a step gradient of petroleum ether and acetone $(0 \%-100 \%, \mathrm{v} / \mathrm{v})$. Fraction 6-6 was separated into five fractions on a Sephadex LH-20 column using a mixture of $\mathrm{MeOH}$ and $\mathrm{CHCl}_{3}(1: 1, \mathrm{v} / \mathrm{v})$ as the eluent. Fraction 6-6-2 was further purified by semipreparative HPLC with $70 \% \mathrm{MeOH}$ to give $1\left(8.1 \mathrm{mg}, t_{\mathrm{R}} 12.8 \mathrm{~min}\right)$. Fraction 6-7 was also purified over a Sephadex LH-20 column eluting with a mixture of $\mathrm{MeOH}$ and $\mathrm{CHCl}_{3}(1: 1, \mathrm{v} / \mathrm{v})$ and $100 \% \mathrm{MeOH}$, before being further purified by semipreparative HPLC with $50 \% \mathrm{MeOH}$ to give compounds 3 (11.4 mg, $t_{\mathrm{R}} 11.2 \mathrm{~min}$ ) and $d l-4$ ( $\left.3 \mathrm{mg}, t_{\mathrm{R}} 12.9 \mathrm{~min}\right)$. The racemic $d l-4$ was purified over a Chiralpak IC column eluting with n-hexane and $\mathrm{EtOH}(20: 80, \mathrm{v} / \mathrm{v})$ to give the pure compounds $\mathbf{4 a}\left(0.8 \mathrm{mg}, t_{\mathrm{R}} 15.9 \mathrm{~min}\right)$ and $\mathbf{4 b}(0.8 \mathrm{mg}$, $t_{\mathrm{R}} 30.2 \mathrm{~min}$ ). Fraction 7 was purified by CC over silica gel using a step gradient elution with petroleum ether and acetone $(0 \%-100 \%, v / v)$ to give five fractions (fraction 7-1 to 7-5). Fraction 7-5 was also purified over a Sephadex LH-20 column eluting with $\mathrm{MeOH}$ and $\mathrm{CHCl}_{3}(1: 1, \mathrm{v} / \mathrm{v})$, before being further purified by semipreparative HPLC with $60 \% \mathrm{MeOH}$ to give $2\left(4.0 \mathrm{mg}, t_{\mathrm{R}} 10.2 \mathrm{~min}\right)$.

$(d l)$-Epoxy-11-deoxyfunicone $(d l-4)$ : colorless needles, mp $166{ }^{\circ} \mathrm{C}$ (hexane-EtOAc), IR ( $\left.\mathrm{KBr}\right) v_{\max }$ 1731, 1670, 1649, 1646, 1363, 1216, 1154, 1058 and $905 \mathrm{~cm}^{-1}$; HRESI-MS m/z $397.0902[\mathrm{M}+\mathrm{Na}]^{+}$. ${ }^{1} \mathrm{H}$ NMR (600 MHz) and ${ }^{13} \mathrm{C}$ NMR (150 MHz) see Table S1.

(9R,14S)-Epoxy-11-deoxyfunicone (4a): $[\alpha]^{23}{ }_{\mathrm{D}}+15.8(c$ 0.04, $\mathrm{MeOH}) ; \mathrm{UV}(\mathrm{MeOH}) \lambda_{\max }(\log \varepsilon)$ 208 (4.18), $238(3.90)$ and $286(4.03) \mathrm{nm} ; \mathrm{CD}(c 0.04, \mathrm{MeOH}) \lambda_{\max }(\Delta \varepsilon) 212(+3.1), 280(-7.4)$ and $336(+6.1) \mathrm{nm}$. 
(9S,14R)-Epoxy-11-deoxyfunicone (4b): $[\alpha]^{23}{ }_{\mathrm{D}}-15.4(c$ 0.04, $\mathrm{MeOH}) ; \mathrm{UV}(\mathrm{MeOH}) \lambda_{\max }(\log \varepsilon)$ 208 (4.18), $238(3.90)$ and $286(4.03) \mathrm{nm} ; \mathrm{CD}(c 0.04, \mathrm{MeOH}) \lambda_{\max }(\Delta \varepsilon) 212(-2.9), 280(+7.8)$ and $336(-5.8) \mathrm{nm}$.

$\mathrm{X}$-ray crystal data for $(d l)$-epoxy-11-deoxyfunicone ( $d l$-4): Racemic $d l$-4 was obtained as a colorless orthorhombic crystal from a mixture of $\mathrm{MeOH}$ and $\mathrm{CHCl}_{3}(1: 1, \mathrm{v} / \mathrm{v})$ : molecular formula $\mathrm{C}_{19} \mathrm{H}_{18} \mathrm{O}_{8}$; space group $P 2{ }_{1}{ }_{1} 2_{1}$ with $a=7.8524(10) \AA, b=15.0361(2) \AA, c=15.2591(2) \AA, V=1801.63(4) \AA^{3}$, $Z=4, D_{\text {calcd. }}=1.380 \mathrm{mg} / \mathrm{m}^{3}, \mu=0.922 \mathrm{~mm}^{-1}$, and $F(000)=784$; crystal size: $0.60 \times 0.40 \times 0.40 \mathrm{~mm}^{3}$; Absolute structure parameter: $0.25(13) . T=150(2) \mathrm{K}$. A total of 16,620 unique reflections $\left(2 \theta<50^{\circ}\right)$ were collected on a CrysAlis PRO CCD area detector diffractometer with graphite-monochromated $\mathrm{Cu} \mathrm{K \alpha}$ radiation $(\lambda=1.54184 \AA)$. The structure was solved by direct methods (SHELXS-97) and expanded using Fourier techniques (SHELXL-97). The final cycle of full-matrix least-squares refinement was based on 3401 unique reflections $\left(2 \theta<50^{\circ}\right)$ and 248 variable parameters and converged with unweighted and weighted agreement factors of R1 $=0.0288$ and $\mathrm{R} 2=0.0736$ for I $>2 \sigma(I)$ data. Crystallographic data for $d l-4$ have been deposited with the Cambridge Crystallographic Data Centre (No. CCDC 856489). Copies of the data can be obtained, free of charge, on application to the CCDC, 12 Union Road, Cambridge CB2 1EZ, UK.

\subsection{Bioassay}

The cytotoxic effects of the compounds on HL-60 and H975 tumor cells were evaluated using the MTT assay method [21]. Briefly, the cell lines were grown in RPMI-1640 medium supplemented with $10 \%$ FBS under a humidified atmosphere of $5 \% \mathrm{CO}_{2}$ and $95 \%$ air at $37{ }^{\circ} \mathrm{C}$. Cell suspensions of $200 \mu \mathrm{L}$ in size at a density of $5 \times 10^{4}$ cell $\mathrm{mL}^{-1}$ were plated in 96-well microtiter plates and incubated for $24 \mathrm{~h}$ at $37{ }^{\circ} \mathrm{C}$. Portions of the test solutions $(2 \mu \mathrm{L}$ in $\mathrm{MeOH})$ were then added to each well and the resulting mixtures were incubated for $72 \mathrm{~h}$. MTT solution $(20 \mu \mathrm{L}, 5 \mathrm{mg} / \mathrm{mL}$ in IPMI-1640 medium) was then added to each well, and the resulting mixtures were incubated for $4 \mathrm{~h}$. The old media containing the MTT $(150 \mu \mathrm{L})$ were then gently replaced with DMSO and pipetted to allow for the dissolution of the formazan crystals. The absorbance values were then determined on a SpectraMax Plus plate reader at $540 \mathrm{~nm}$. The $\mathrm{IC}_{50}$ values were calculated as the concentration of compound required to reduce cell viability by $50 \%$.

\section{Conclusions}

In contrast to the discrete fungal and bacterial controls, the co-fermentation of Penicillium sp. WC-29-5 with Streptomyces fradiae 007 led to the production of four completely aromatic polyketides, including deoxyfunicone (1), 1,3,8-trihydroxy-6-methylxanthen-9-one (2), (9R,14S)-epoxy-11-deoxy funicone (4a) and $(9 S, 14 R)$-epoxy-11-deoxyfunicone (4b). Furthermore, the absolute configurations of compounds $\mathbf{4 a}$ and $\mathbf{4 b}$ as well as their cytotoxicities against HL-60 and H1975 cells have been reported in this study for the first time. The results of the current study demonstrate that the co-culturing of two microbes can induce the synthesis of secondary metabolites that would otherwise not be formed during the cultivation of the same species in isolation. Furthermore, the microorganisms used in the current study survived in a competitive environment and produced bioactive secondary metabolites. 


\section{Acknowledgments}

This work was supported by grants from NSFC (Nos. 21172204, 41376148 \& 81373298), from 973 of China (No. 2010CB833804), and from 863 Program of China (Nos. 2013AA092901 \& 2012AA092104), and from the Special Fund for Marine Scientific Research in the Public Interest of China (No. 2010418022-3).

\section{Author Contributions}

The structure identification and the paper writing were finished by Y. Wang. The racemic mixture was separated by L. Wang. The co-culture was performed the compounds were isolated by Y. Zhuang. The ECD calculations of the compounds were finished by F. Kong. X-ray crystal data were measured and analyzed by C. Zhang. And W. Zhu was responsible for the experimental design, the paper revision and the fund applications.

\section{Conflicts of Interest}

The authors declare no conflict of interest.

\section{References}

1. Cragg, G.M.; Grothaus, P.G.; Newman, D.J. Impact of natural products on developing new anti-cancer agents. Chem. Rev. 2009, 109, 3012-3043.

2. Nett, M.; Ikeda, H.; Moore, B.S. Genomic basis for natural product biosynthetic diversity in the actinomycetes. Nat. Prod. Rep. 2009, 26, 1362-1384.

3. Brakhage, A.A.; Schroeckh, V. Fungal secondary metabolites strategies to activate silent gene clusters. Fungal Genet. Biol. 2011, 48, 15-22.

4. Winter, J.M.; Behnken, S.; Hertweck, C. Genomics-inspired discovery of natural products. Curr. Opin. Chem. Biol. 2011, 15, 22-31.

5. Brakhage, A.A. Regulation of fungal secondary metabolism. Nat. Rev. Microbiol. 2013, 11, 21-32.

6. Scherlach, K.; Hertweck, C. Triggering cryptic natural product biosynthesis in microorganisms. Org. Biomol. Chem. 2009, 7, 1753-1760.

7. Marmann, A.; Aly, A.H.; Lin, W.; Wang, B.; Proksch, P. Co-cultivation-A powerful emerging tool for enhancing the chemical diversity of microorganisms. Mar. Drugs 2014, 12, 1043-1065.

8. Wang, Y.; Zheng, J.; Liu, P.; Wang, W.; Zhu, W. Three new compounds from Aspergillus terreus PT06-2 grown in a high salt medium. Mar. Drugs 2011, 9, 1368-1378.

9. Wang, Y.; Lu, Z.; Sun, K.; Zhu, W. Effects of high salt stress on secondary metabolites from marine-derived fungus Spicaria elegans. Mar. Drugs 2011, 9, 535-542.

10. Wang, P.; Xi, L.; Liu, P.; Wang, Y.; Wang, W.; Huang, Y.; Zhu, W. Diketopiperazine derivatives from the marine-derived actinomycete Streptomyces sp. FXJ7.328. Mar. Drugs 2013, 11, 1035-1049.

11. Woodyer, R.D.; Shao, Z.; Thomas, P.M.; Kelleher, N.L.; Blodgett, J.A.; Metcalf, W.W.; van der Donk, W.A.; Zhao, H. Heterologous production of fosfomycin and identification of the minimal biosynthetic gene cluster. Chem. Biol. 2006, 13, 1171-1182. 
12. Huang, F.; Haydock, S.F.; Mironenko, T.; Spiteller, D.; Li, Y.; Spencer, J.B. The neomycin biosynthetic gene cluster of Streptomyces fradiae NCIMB 8233: Characterisation of an aminotransferase involved in the formation of 2-deoxystreptamine. Org. Biomol. Chem. 2005, 3, 1410-1418.

13. Zhuang, Y.B.; Wang, Y.; Liu, P.P.; Zhu, W.M. Compound mutation breeding of the staurosporine producing strains. Chin. J. Mar. Drugs 2011, 30, 29-33.

14. Fu, P.; Zhuang, Y.; Wang, Y.; Liu, P.; Qi, X.; Gu, K.; Zhang, D.; Zhu, W. New indolocarbazoles from a mutant strain of the marine-derived actinomycete Streptomyces fradiae 007M135. Org. Lett. 2012, 14, 6194-6197.

15. Sassa, T.; Nukina, M.; Suzuki, Y. Deoxyfunicone, a new gamma-pyrone metabolite form a resorcylide-producing fungus (Penicillium sp.). Agric. Biol. Chem. 1991, 55, 2415-2416.

16. Koch, K.; Podlech, J.; Pfeiffer, E.; Metzler, M. Total synthesis of alternariol. J. Org. Chem. 2005, 70, 3275-3276.

17. Komai, S.; Hosoe, T.; Itabashi, T.; Nozawa, K.; Okada, K.; Campos Takaki, G.M.; Chikamori, M.; Yaguchi, T.; Fukushima, K.; Miyaji, M.; et al. A new funicone derivative isolated from Talaromyces flavus IFM52668. Mycotoxins 2004, 54, 15-19.

18. Stephens, P.J.; Pan, J.J.; Krohn, K.J. Determination of the absolute configurations of pharmacological natural products via density functional theory calculations of vibrational circular dichroism: the new cytotoxic iridoid prismatomerin. J. Org. Chem. 2007, 72, 7641-7649.

19. Harada, N.; Nakanishi, K. Circular Dichroic Spectroscopy: Exciton Coupling in Organic Stereochemistry; University Sciences Books: Mill Valley, CA, USA, 1983.

20. Jiao, W.H.; Huang, X.J.; Yang, J.S.; Yang, F.; Piao, S.J.; Gao, H.; Li, J.; Ye, W.C.; Yao, X.S.; Chen, W.S.; et al. Dysidavarones A-D, new sesquiterpene quinones from the marine sponge Dysidea avara. Org. Lett. 2012, 14, 202-205.

21. Mosmann, T. Rapid colorimetric assay for cellular growth and survival: Application to proliferation and cytotoxicity assays. J. Immunol. Methods 1983, 65, 55-63.

(C) 2014 by the authors; licensee MDPI, Basel, Switzerland. This article is an open access article distributed under the terms and conditions of the Creative Commons Attribution license (http://creativecommons.org/licenses/by/3.0/). 